\title{
On the Cramer-Rao Bound for Carrier Frequency Estimation in the Presence of Phase Noise
}

\author{
Alan Barbieri, Student Member, IEEE, and Giulio Colavolpe
}

\begin{abstract}
We consider the carrier frequency offset estimation in a digital burst-mode satellite transmission affected by phase noise. The corresponding Cramer-Rao lower bound is analyzed for linear modulations under a Wiener phase noise model and in the hypothesis of knowledge of the transmitted data. Even if we resort to a Monte Carlo average, from a computational point of view the evaluation of the Cramer-Rao bound is very hard. We introduce a simple but very accurate approximation that allows to carry out this task in a very easy way. As it will be shown, the presence of the phase noise produces a remarkable performance degradation of the frequency estimation accuracy. In addition, we provide asymptotic expressions of the CramerRao bound, from which the effect of the phase noise and the dependence on the system parameters of the frequency offset estimation accuracy clearly result. Finally, as a by-product of our derivations and approximations, we derive a couple of estimators specifically tailored for the phase noise channel that will be compared with the classical Rife and Boorstyn algorithm, gaining in this way some important hints on the estimators to be used in this scenario.
\end{abstract}

Index Terms-Cramer-Rao bound, Frequency estimation, Phase noise.

\section{INTRODUCTION}

$\mathbf{T}$ HE Cramer-Rao bound (CRB) is a fundamental lower limit to the variance of any unbiased parameter estimator [1]. As such, it gives the ultimate accuracy that can be achieved in synchronization operations.

For the frequency offset estimation problem, this bound was computed under different assumptions. The CRBs for the frequency estimation of a single tone in the case of both a known and an unknown constant phase were computed in [2] based on a discrete-time observation model. These results can be also directly applied to the case of phaseshift keying (PSK) signals when transmitted data are perfectly known, i.e., when a data-aided (DA) frequency estimation is performed based on a known preamble. The CRBs in the case of non-data aided (NDA) operations for binary and quaternary PSK (BPSK and QPSK) were derived in [3] and extended to quadrature amplitude modulations (QAM) in [4]. In these papers, the phase offset was assumed known or the case of joint phase and frequency estimation was considered. Finally, for PSK signals, in [5] the CRBs for DA and NDA estimators

Manuscript received April 18, 2005; revised September 5, 2005; accepted January 3, 2006. The associate editor coordinating the review of this paper and approving it for publication was $\mathrm{H}$. Li. This paper was presented in part at the IEEE Global Telecommunications Conference (GLOBECOM'05), St. Louis, MO, U.S.A., November-December 2005. This work is funded by the European Space Agency, ESA-ESTEC, Noordwijk, The Netherlands.

The authors are with Università di Parma, Dipartimento di Ingegneria dell'Informazione, Parco Area delle Scienze 181A, I-43100 Parma, Italy (email: barbieri@tlc.unipr.it, giulio@unipr.it).

Digital Object Identifier 10.1109/TWC.2007.05270. considering both the case of unknown phase offset uniformly distributed in the interval $[0,2 \pi)$ and the case of joint phase and frequency estimation were computed. The comparison between the discrete-time model commonly used and the true continuous-time model was discussed in [5], showing that, although the correct observation model yields the smaller $\mathrm{CRB}$, the difference between the CRBs resulting from the two models is apparent only at very low values of the signal-tonoise ratio (SNR).

All these papers, as well as the papers dealing with the algorithms for frequency estimation (see for example [2], [6]-[9], or [10] and references therein) refer to an idealized situation in which the phase offset is constant. However, in modern burst-mode satellite communications, it is common to incur in a strong time-varying phase noise due to the oscillator instabilities. In this case, it is interesting to quantify the resulting performance degradation in frequency estimation operations. To this purpose, we consider the case of a burst-mode transmission using a linearly modulated signal. In this scenario, it is usual to have a first coarse carrier frequency acquisition to reduce the frequency error followed, after timing recovery, by a fine DA frequency estimator based on a known preamble [11]. Phase estimation and tracking is then performed after frequency compensation. Since we are interested in the operations of the fine DA frequency estimator, we consider this setting: known data, ideal timing, and a discrete observation model. In addition, the phase noise has to be considered as a nuisance parameter, being estimated after frequency compensation.

The computation of the resulting CRB is a formidable task. In fact, the likelihood function necessary for the CRB computation must be obtained by averaging over the phase noise. A closed-form expression does not exist and even if we resort to numerical methods, the computational effort is very hard. In this paper, we introduce a simple but very accurate closed form for the likelihood function and then we perform the expectation necessary to obtain the CRB by means of an arithmetical average over a number of computer-generated received samples. The result is in perfect agreement with the closed-form asymptotic expressions of the CRB that we also compute in this paper. The derived approximated likelihood function can be also employed to derive new estimation algorithms and to gain new hints on the existing algorithms tailored for a constant phase offset.

The reminder of this paper is organized as follows. Section II introduces the channel model and the definition of the CRB. In Section III, an approximated closed-form expression of the likelihood function necessary for the CRB compu- 
tation is described along with its asymptotic expressions. The computation of the CRB is faced in Section IV. Using the approximated closed-form expression of the likelihood function, the CRB computation through a Monte Carlo average becomes a very easy task. We also compute the exact closedform expressions of the CRB for low and high values of the signal-to-noise ratio. The approximated and asymptotic closed-form expressions of the likelihood function, computed in Section III, are also used in Section V to derive new estimation algorithms for this scenario. Finally, in Section VI we present some numerical results and in Section VII we point out some concluding remarks.

\section{SYSTEM MODEL AND THE CRB}

We consider the transmission of a sequence of complex modulation symbols $\left\{a_{k}\right\}_{k=0}^{K-1}$, belonging to an $M$-ary constellation of unit average energy, over an additive white Gaussian noise (AWGN) channel affected by carrier phase noise and a constant frequency offset $\nu$. Symbols $a_{k}$ are linearly modulated. Assuming Nyquist transmitted pulses, matched filtering, a small frequency offset and phase variations slow enough so as no intersymbol interference arises, the discrete-time baseband received signal is given by

$$
r_{k}=a_{k} e^{j\left(2 \pi \nu k T+\theta_{k}\right)}+w_{k}, \quad k=0,1, \ldots, K-1
$$

where $T$ is the symbol interval and the noise samples $\left\{w_{k}\right\}_{k=0}^{K-1}$ are independent and identically distributed (i.i.d.), complex, circularly symmetric Gaussian random variables (rvs), each with mean zero and variance equal to $2 \sigma^{2}=$ $N_{0} / E_{S}, N_{0}$ being the one-sided noise power spectral density and $E_{S}$ the received signal energy per information symbol. For the time-varying channel phase $\theta_{k}$, we assume a randomwalk (Wiener) model widely used in the literature (as an example, see [12]-[17]) since it is a good approximation of more involved models:

$$
\theta_{k+1}=\theta_{k}+\Delta_{k}
$$

in which $\left\{\Delta_{k}\right\}$ are real i.i.d. Gaussian rvs with mean zero and standard deviation $\sigma_{\Delta}$, and the rv $\theta_{0}$ is uniformly distributed. The rvs $\left\{\theta_{k}\right\}$ are supposed unknown to the receiver, and statistically independent of symbols and noise. When $\sigma_{\Delta}=0$ we obtain the classical case of a constant and uniformly distributed phase offset. Hence, this model has also the clear advantage that it is characterized by a single parameter $\left(\sigma_{\Delta}\right)$ which allows to effectively tune its strength.

Some of the information symbols in the transmitted burst are known to the receiver (pilot symbols) and we assume that the frequency estimation is based on these symbols. For generality, we assume that the inserted $N$ pilot symbols are $\left\{a_{k(n)}\right\}$, where $\{k(n) \mid 0 \leq n \leq N-1\}$ is an index set for the sample times. These symbols and the corresponding received and phase samples are collected into three vectors $\mathbf{a} \triangleq\left\{a_{k(n)}\right\}_{n=0}^{N-1}, \mathbf{r} \triangleq\left\{r_{k(n)}\right\}_{n=0}^{N-1}$, and $\boldsymbol{\theta} \triangleq\left\{\theta_{k(n)}\right\}_{n=0}^{N-1}$.

The CRB for this estimation problem is defined as [1]

$$
C R B_{\nu}^{-1}=E_{\mathbf{r}}\left[-\frac{\partial^{2}}{\partial \nu^{2}} \ln p(\mathbf{r} \mid \nu)\right]
$$

where $p(\mathbf{r} \mid \nu)$ is the probability density function (pdf) of $\mathbf{r}$ given $\nu$, the derivative is evaluated at the true value of $\nu$, and
$E_{\mathbf{r}}$ denotes statistical expectation with respect to the vector $\mathbf{r}$. The pdf $p(\mathbf{r} \mid \nu)$ can be obtained as

$$
p(\mathbf{r} \mid \nu)=E_{\boldsymbol{\theta}}\{p(\mathbf{r} \mid \boldsymbol{\theta}, \nu)\}=\int p(\mathbf{r} \mid \boldsymbol{\theta}, \nu) p(\boldsymbol{\theta}) d \boldsymbol{\theta} .
$$

As already mentioned, the likelihood function $p(\mathbf{r} \mid \nu)$ cannot be expressed in a closed form. On the other hand, if the expectation in (3) can be easily performed by means of a Monte Carlo average, the computational effort required by the numerical evaluation of the expectation in (4) is much more intensive. In the next section, we describe an approximate but very accurate closed-form expression for this pdf.

In the technical literature, there is an alternative lower bound on the estimator error variance, the so-called modified CRB (MCRB) [18], easy to compute but in general quite looser. For the problem at hand, this bound is not useful. In fact, it can be easily shown that the MCRB has always the same value, independently of the value of $\sigma_{\Delta}$. In other words, the MCRB for $\sigma_{\Delta}=0$ (absence of phase noise) is the same of the MCRB for $\sigma_{\Delta} \neq 0$. Hence, it is not able to describe the effects of the phase noise.

\section{The LiKelihood FunCtion}

In this section, we introduce an approximated closed-form expression for the pdf $p(\mathbf{r} \mid \nu)$ that will be used in the computation of the CRB, and also a couple of asymptotic closed-form expressions, in the absence of phase noise $\left(\sigma_{\Delta}=0\right)$ and in the absence of thermal noise $(\sigma=0)$, respectively.

\section{A. Approximated Closed-Form Expression}

Let us denote by $g\left(\eta, \rho^{2} ; x\right)$ a Gaussian distribution in $x$, with mean value $\eta$ and variance $\rho^{2}$, and by $t(\zeta ; x)$ a Tikhonov distribution in $x$ characterized by the complex parameter $\zeta$, i.e.,

$$
\begin{gathered}
g\left(\eta, \rho^{2} ; x\right)=\frac{1}{\sqrt{2 \pi \rho^{2}}} e^{-\frac{(x-\eta)^{2}}{2 \rho^{2}}} \\
t(\zeta ; x)=\frac{1}{2 \pi \mathrm{I}_{0}(|\zeta|)} e^{\operatorname{Re}\left[\zeta e^{-j x}\right]}
\end{gathered}
$$

where $\mathrm{I}_{0}(x)$ is the zero-th order modified Bessel function of the first kind. By using these definitions and taking into account the system model (1), we may express, discarding irrelevant proportionality factors independent of $\boldsymbol{\theta}$ and $\nu$

$$
\begin{aligned}
p(\mathbf{r} \mid \boldsymbol{\theta}, \nu) & =\prod_{n=0}^{N-1} p\left(r_{k(n)} \mid \theta_{k(n)}, \nu\right) \\
& \propto \prod_{n=0}^{N-1} \exp \left\{\frac{1}{\sigma^{2}} \operatorname{Re}\left[r_{k(n)} a_{k(n)}^{*} e^{-j\left(2 \pi \nu k(n) T+\theta_{k(n)}\right)}\right]\right\} \\
& =\prod_{n=0}^{N-1} t\left(z_{k(n)} ; \theta_{k(n)}\right) \\
p(\boldsymbol{\theta}) & =p\left(\theta_{k(0)}\right) \prod_{n=1}^{N-1} p\left(\theta_{k(n)} \mid \theta_{k(n-1)}\right)
\end{aligned}
$$

having defined $z_{k} \triangleq \frac{r_{k} a_{k}^{*}}{\sigma^{2}} e^{-j 2 \pi \nu k T}$. In (6b), the pdf $p\left(\theta_{k(0)}\right)$ is $p\left(\theta_{k(0)}\right)=1 / 2 \pi$, since the rv $\theta_{k(0)}$ is uniformly distributed. According to the phase noise model (2), the increment $\theta_{k(n)}-\theta_{k(n-1)}$ is Gaussian with standard deviation 
$\rho(n)=\sigma_{\Delta} \sqrt{k(n)-k(n-1)}$. However, since the channel phase is defined modulo $2 \pi$, from a conceptual viewpoint, the pdf $p\left(\theta_{k(n)} \mid \theta_{k(n-1)}\right)$ is the pdf of the increment $\theta_{k(n)}-$ $\theta_{k(n-1)} \bmod 2 \pi$, i.e.,

$$
\begin{aligned}
p\left(\theta_{k(n)} \mid \theta_{k(n-1)}\right) & =\sum_{m=-\infty}^{\infty} g\left(\theta_{k(n-1)}, \rho^{2}(n) ; \theta_{k(n)}-2 \pi m\right) \\
& =\sum_{m=-\infty}^{\infty} g\left(2 \pi m, \rho^{2}(n) ; \theta_{k(n)}-\theta_{k(n-1)}\right)
\end{aligned}
$$

in any interval of length $2 \pi$. We assume, for the adopted pilot distribution, $\rho(n) \ll 2 \pi$. Under this hypothesis, in the interval $\left[\theta_{k(n-1)}-\pi, \theta_{k(n-1)}+\pi\right)$, in practice only the term with $m=0$ in (7) gives a non negligible contribution. Hence, in the interval $\left[\theta_{k(n-1)}-\pi, \theta_{k(n-1)}+\pi\right)$ we may approximate

$$
\begin{aligned}
p\left(\theta_{k(n)} \mid \theta_{k(n-1)}\right) & \simeq g\left(\theta_{k(n-1)}, \rho^{2}(n) ; \theta_{k(n)}\right) \\
& =g\left(0, \rho^{2}(n) ; \theta_{k(n)}-\theta_{k(n-1)}\right)
\end{aligned}
$$

i.e., in this interval the pdf $p\left(\theta_{k(n)} \mid \theta_{k(n-1)}\right)$ is practically Gaussian with mean $\theta_{k(n-1)}$ and standard deviation $\rho(n)$. Since all the integrals involved in the following derivation can be defined in any interval of length $2 \pi$, for convenience we will consider the interval $\left[\theta_{k(n-1)}-\pi, \theta_{k(n-1)}+\pi\right)$.

By substituting (6a) and (6b) into (4), observing that

$$
t(z ; \theta) t(u ; \theta)=\frac{\mathrm{I}_{0}(|z+u|)}{2 \pi \mathrm{I}_{0}(|z|) \mathrm{I}_{0}(|u|)} t(z+u ; \theta)
$$

and using the following approximation [19], [20] ${ }^{1}$

$$
\int t(\zeta, x) g\left(x, \rho^{2} ; y\right) d x \simeq t\left(\frac{\zeta}{1+\rho^{2}|\zeta|} ; y\right)
$$

discarding irrelevant multiplicative terms, after some manipulations (see Appendix I) we obtain the following expression of the likelihood function:

$$
p(\mathbf{r} \mid \nu) \approx \prod_{n=0}^{N-2} \frac{\mathrm{I}_{0}\left(\left|z_{k(n)}+u_{n}\right|\right)}{\mathrm{I}_{0}\left(\left|u_{n}\right|\right)}
$$

where coefficients $u_{n}, n=N-2, \ldots, 0$, can be recursively computed as

$$
u_{n}=\frac{u_{n+1}+z_{k(n+1)}}{1+[k(n+1)-k(n)] \sigma_{\Delta}^{2}\left|u_{n+1}+z_{k(n+1)}\right|}
$$

with initial condition $u_{N-1}=0$.

\section{B. Absence of Phase Noise}

When $\sigma_{\Delta}=0$, i.e., when a constant unknown phase offset is considered, we obtain an exact expression for the likelihood function which is equivalent to that derived in [5]. In fact, in this case (10) holds with equality and coefficients $u_{n}$ can be expressed as

$$
u_{n}=\sum_{\ell=n+1}^{N-1} z_{k(\ell)}
$$

\footnotetext{
${ }^{1}$ Note that, when $\rho=0,(10)$ holds with equality. By numerical integration, we verified that the mean square error between the left and right hand side of (9) is always less than $10^{-3}$ for $\rho \leq 30$ degrees.
}

Hence, we have

$$
p(\mathbf{r} \mid \nu) \propto \prod_{n=0}^{N-2} \frac{\mathrm{I}_{0}\left(\left|\sum_{\ell=n}^{N-1} z_{k(\ell)}\right|\right)}{\mathrm{I}_{0}\left(\left|\sum_{\ell=n+1}^{N-1} z_{k(\ell)}\right|\right)} .
$$

\section{Absence of Thermal Noise}

We now consider the case of absence of thermal noise (i.e., $\sigma=0)$. This is an approximation of the case when the SNR is large enough so as the effect of thermal noise is negligible with respect to phase noise.

In the next sections, it will be shown that the likelihood function is always periodic with a period depending on the pilot distribution. In the case of absence of thermal noise, through the manipulations detailed in Appendix II, we find that, in an interval around each maximum, the pdf $p(\mathbf{r} \mid \nu)$ can be expressed as

$$
\begin{aligned}
& p(\mathbf{r} \mid \nu) \propto \exp \left\{-\frac{(2 \pi)^{2} D}{2 \sigma_{\Delta}^{2}}[\nu T\right. \\
& \left.\left.-\frac{1}{2 \pi D} \sum_{n=1}^{N-1} \arg \left(r_{k(n)} a_{k(n)}^{*} r_{k(n-1)}^{*} a_{k(n-1)}\right)\right]^{2}\right\}
\end{aligned}
$$

having defined $D=k(N-1)-k(0)$. Hence, in this case, around each maximum the likelihood function is Gaussian and does not depend on the number and position of pilot symbols, but only on the distance $D$ between the first and the last pilot symbol.

\section{THE CRAMER-RAO BOUND}

We now describe the computation of the CRB for the problem at hand.

As already mentioned, a first computationally intensive method is based on a numerical evaluation, through Monte Carlo average, of both the expectations in (3) and (4). A stepby-step discussion on how to implement this "full" Monte Carlo average is reported in Appendix III. The corresponding result, denoted as $C R B_{M C}$, is used to verify the accuracy of the CRB obtained through the use of the simplified approximated closed-form expression (11) of the likelihood function and denoted as $C R B_{\text {simp }}$. In this latter case, the Monte Carlo average is only used to compute the expectation in (3). In the case of absence of phase noise, by using the closed-form expression (14), the $C R B_{\text {simp }}$ is exact. Hence, the proposed method gives the exact $\mathrm{CRB}$ for the case of known data, a constant and unknown phase offset, and the discrete-time observation model.

The low- and high-SNR limits of the CRB can be also computed in closed form. By observing that for low SNR values the arguments of the Bessel functions in (11) assume low values, we can use the limiting form for small arguments $\ln \mathrm{I}_{0}(x) \simeq x^{2} / 4$, obtaining (see Appendix IV for the details)

$$
C R B_{L}=\frac{\sigma^{4}}{2 \pi^{2} T^{2} \sum_{n=0}^{N-2} \sum_{\ell=n+1}^{N-1} F(n, \ell)}
$$


where

$$
F(n, \ell) \triangleq[k(\ell)-k(n)]^{2}\left|a_{k(l)}\right|^{2}\left|a_{k(n)}\right|^{2} e^{-\frac{1}{2}[k(\ell)-k(n)] \sigma_{\Delta}^{2}} .
$$

For $\sigma_{\Delta}=0$, PSK signals, and $N$ consecutive pilots, i.e., $k(n)=n, n=0,1, \ldots, N-1$, this result coincides with the low-SNR limit in [5]. For $\sigma_{\Delta}>0$, the CRB increases and this means that there is a performance degradation due to the phase noise.

For high SNR values, by using (15) in (3) we obtain

$$
C R B_{H}=\frac{1}{D T^{2}}\left(\frac{\sigma_{\Delta}}{2 \pi}\right)^{2} \text {. }
$$

This high-SNR limit allows to draw some important considerations. First of all, in the presence of a time-varying phase, the CRB has a floor, i.e., it is not possible to reach the desired estimation accuracy simply increasing the SNR value. In addition, the asymptotic CRB only depends on the positions of the first and last pilot symbol (as the asymptotic likelihood function (15)) and is completely independent of the actual pilot distribution. Let us now consider the particular pilot distribution characterized by $k(n)=n L$, where $L \geq 1$ is an integer constant which plays the role of the distance between two consecutive pilot symbols (hence, $L=1$ depicts the situation of $N$ consecutive pilot symbols). In this case, being $D=(N-1) L$, the high-SNR limit assumes the form

$$
C R B_{H}=\frac{1}{(N-1) L T^{2}}\left(\frac{\sigma_{\Delta}}{2 \pi}\right)^{2} .
$$

In [18] it has been demonstrated that, for a large enough SNR and a constant phase offset, the CRB coincides with the MCRB. It can be straightforwardly shown that, with the considered pilot distribution, the MCRB takes on value

$$
M C R B=\frac{3 N_{0}}{2 \pi^{2} N^{3} L^{2} E_{S} T^{2}}
$$

(see also [21] for the effect of a non uniform pilot distribution on the likelihood function). Hence, for high SNR values, the CRB goes as $N^{-1}$ in the presence of phase noise whereas it goes as $N^{-3}$ for a constant phase offset. As a consequence, an increase in the estimation window has still a beneficial effect on the estimation accuracy, mitigated by the fact that the presence of a time-varying phase leads to almost independent received samples when the window becomes larger.

Similarly, the CRB goes as $L^{-1}$ for a time-varying phase whereas in (20) it goes as $L^{-2}$ for a constant phase offset. This behavior is due to the fact that increasing the distance between two consecutive pilot symbols has the same effect of increasing the phase noise variance.

\section{Estimation Algorithms}

By using the expressions for the likelihood function derived in Section III we can design a couple of maximum likelihood (ML) estimation algorithms for this scenario. We consider the above mentioned pilot distribution $k(n)=n L$. In this case, for the considered discrete-time signal model, values of frequency offset which differ of $1 / L T$ are indistinguishable since they produce the same received samples $r_{k(n)}=r_{n L}$. Hence, the likelihood functions are $\frac{1}{L}$-periodic with respect to the normalized frequency offset $\nu T$. This means that the valid estimation range must be small enough so as no more than one global maximum appears in the likelihood function, that is, the possible values of the frequency offset must be inside the range $\left[-\frac{1}{2 L T}, \frac{1}{2 L T}\right]$.

By considering the likelihood function (11), we obtain the following estimator

$$
\begin{aligned}
\hat{\nu} & =\underset{\nu}{\operatorname{argmax}} p(\mathbf{r} \mid \nu)=\underset{\nu}{\operatorname{argmax}} \ln p(\mathbf{r} \mid \nu) \\
& =\underset{\nu}{\operatorname{argmax}} \sum_{n=0}^{N-2}\left[\ln \mathrm{I}_{0}\left(\left|z_{n L}+u_{n}\right|\right)-\ln \mathrm{I}_{0}\left(\left|u_{n}\right|\right)\right] .
\end{aligned}
$$

The search for the maximum of the log-likelihood function can be accomplished, as for the Rife and Boorstyn algorithm [2], in two steps. In a first coarse search, the log-likelihood function is evaluated for some values of the frequency offset in the range $\left[-\frac{1}{2 L T}, \frac{1}{2 L T}\right]$ and the value $\nu_{c s}$ which corresponds to the maximum value is obtained. Then, with a fine search the value of $\nu$ closest to $\nu_{c s}$ which maximizes the loglikelihood function is located, for example by using the secant method or a bisection-like algorithm. This estimator will be denoted as $E_{P N}$. Obviously, it is quite complex for a receiver implementation. In the numerical results it will be used as a term of comparison to evaluate the performance that can be achieved by a practical estimator.

Let us now take into account the asymptotic expression of the likelihood function. A ML estimator, based upon (15), is characterized by the following simple estimation rule $(D=$ $(N-1) L$ for the above mentioned pilot distribution)

$$
\hat{\nu}=\frac{\sum_{n=1}^{N-1} \arg \left[r_{n L} a_{n L}^{*} r_{(n-1) L}^{*} a_{(n-1) L}\right]}{2 \pi(N-1) L T}
$$

that is very similar to the Kay estimator except for the weighting coefficients [6], [10]. We will denote this estimator as $E_{\text {asymp }}$. It is straightforward to show that, for high SNR values, this estimator is unbiased.

\section{NumeriCAL RESUlts}

Although the results in the previous sections can be applied to general linear modulations, in the numerical results we consider $M$-PSK signals since in this case the performance is independent of the adopted pilot sequence. We show the accuracy of the CRB computed by using the simplified closedform expression of the likelihood function. The performance of the derived estimators is also shown and compared with the CRB and with the performance of "classical" frequency estimators.

In Fig. 1, for $\sigma_{\Delta}=0,2$, and 6 degrees, we show the $C R B_{\text {simp }}$, together with the derived low- and high-SNR asymptotic expressions, as a function of the SNR in the case of $N=64$ consecutive pilot symbols (hence $L=$ $1)$. The $C R B_{M C}$ is also shown. We may observe that the derived simplified method has a very good accuracy since the $C R B_{\text {simp }}$ coincides with the $C R B_{M C}$. The high-SNR value $C R B_{H}$ is reached for values of $E_{S} / N_{0}$ around $10 \mathrm{~dB}$, whereas the low-SNR asymptotic value $C R B_{L}$, which slightly depends on the value of $\sigma_{\Delta}$, is reached only at very low SNR values. The performance degradation for high SNR values due to the presence of phase noise is significant already for $\sigma_{\Delta}=2$ 


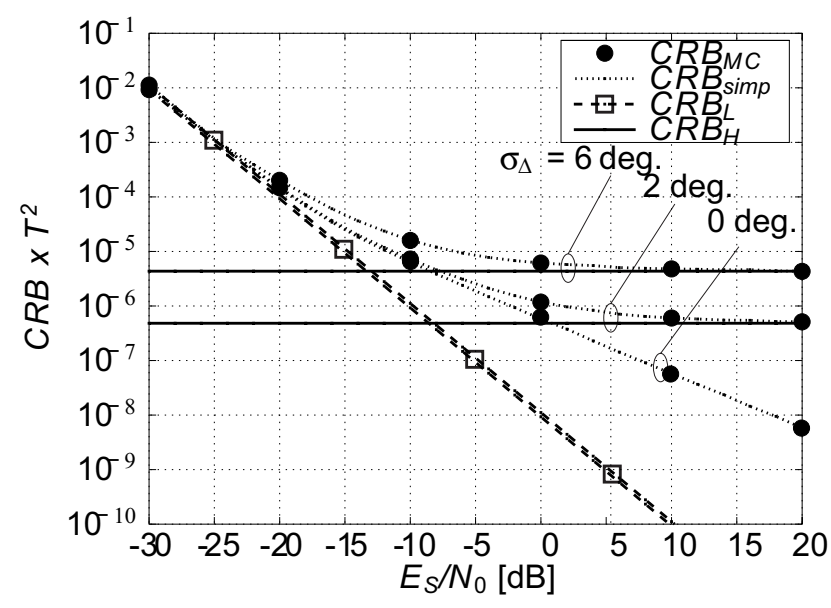

Fig. 1. $\mathrm{CRB}$ in the case of $N=64$ consecutive pilot symbols and different values of $\sigma_{\Delta}$.

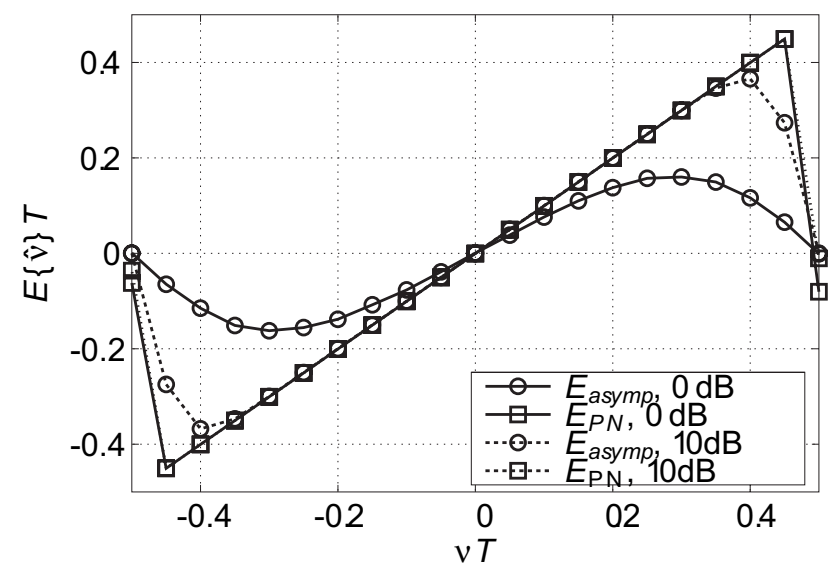

Fig. 2. Mean estimated frequency for $N=64, \sigma_{\Delta}=6$ degrees and two values of $E_{S} / N_{0}$.

degrees, as shown by the presence of the floor predicted by our high-SNR asymptote.

We now consider the performance of the estimators described in the previous section and compare it with the performance of the best algorithm for frequency estimation in the presence of constant phase offset, i.e., the Rife \& Boorstyn $(\mathrm{R} \& \mathrm{~B})$ algorithm [2], denoted in the figures as $E_{R B}$. First of all, in Fig. 2 we show the normalized average estimated frequency $E\{\hat{\nu}\} T$ with respect to the true normalized frequency offset $\nu T$ for the two estimation algorithms $E_{\text {asymp }}$ and $E_{P N}, N=64$ consecutive pilot symbols, a phase noise standard deviation of $\sigma_{\Delta}=6$ degrees, and two different values of $E_{S} / N_{0}$. It is worth noting that, except for the asymptotic estimator working at a low signal-to-noise ratio, the proposed estimators are unbiased in a very large frequency range.

Since we verified that in the considered operating conditions all the estimators are unbiased, we show the estimator variance, normalized to $1 / T^{2}$, which coincides with the mean square estimation error. All the following simulation results have been obtained by generating a random normalized frequency offset in the range $\left[-2 \cdot 10^{-2}, 2 \cdot 10^{-2}\right]$, independently frame by frame.

In Fig. 3, the normalized error variance as a function of

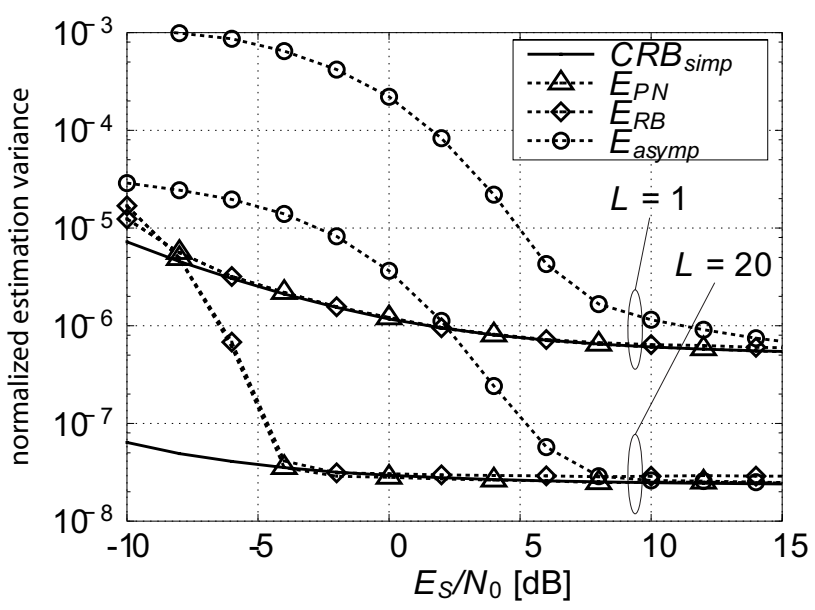

Fig. 3. Normalized estimator variance for $N=64$ and $\sigma_{\Delta}=2$ degrees.

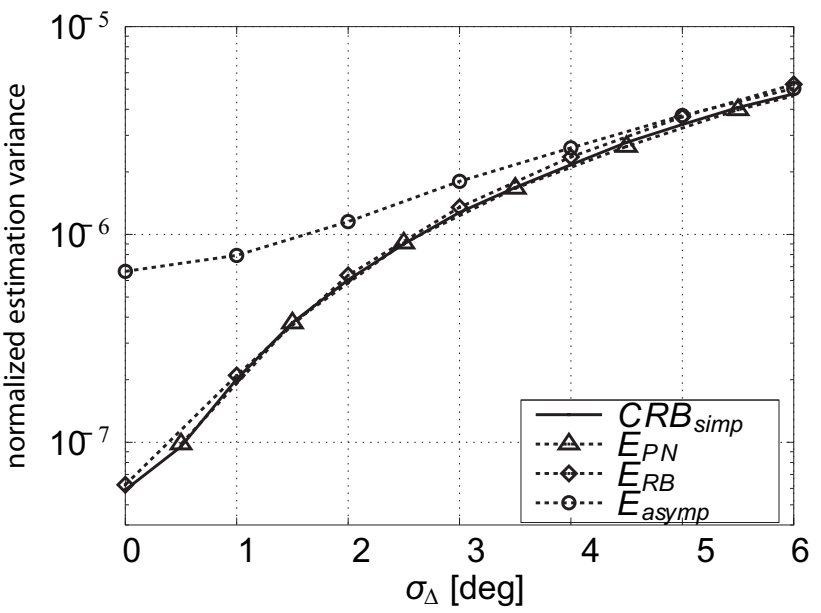

Fig. 4. Normalized estimator variance for $E_{S} / N_{0}=10 \mathrm{~dB}$ and $N=64$ consecutive pilot symbols.

the SNR is shown, for $\sigma_{\Delta}=2$ degrees and $N=64$ pilot symbols. The cases of $L=1$ (consecutive pilots) and $L=$ 20 have been considered. Surprisingly, the R\&B estimator, designed for the case of a constant phase, exhibits only a minor loss with respect to the CRB for high SNR values. ${ }^{2}$ On the other hand, as intuitively expected, estimators $E_{P N}$ and $E_{\text {asymp }}$, designed taking into account the phase noise statistics, are asymptotically optimal. This is a further proof of the correctness of the derivations in Sections III-A and IIIC. At very low SNR values, all the estimators exhibit a larger variance with respect to the bound due to the occurrence of outliers [2], [10] - the corresponding threshold depending on the estimator and on the value of $L$. In particular, the estimator $E_{\text {asymp }}$ has a very high threshold. However, we would like to point out that for $E_{S} / N_{0}$ larger than a few dBs, it is convenient to use the estimator $E_{\text {asymp }}$, which is able to reach the CRB and presents a noticeable smaller complexity with respect to the other estimators.

The performance degradation due to phase noise is highlighted in Fig. 4, where the normalized estimation variance for the considered estimators is reported, together with the

\footnotetext{
${ }^{2}$ We verified that a similar behavior can be observed for other estimation algorithms such as, for example, those described in [7], [8].
} 


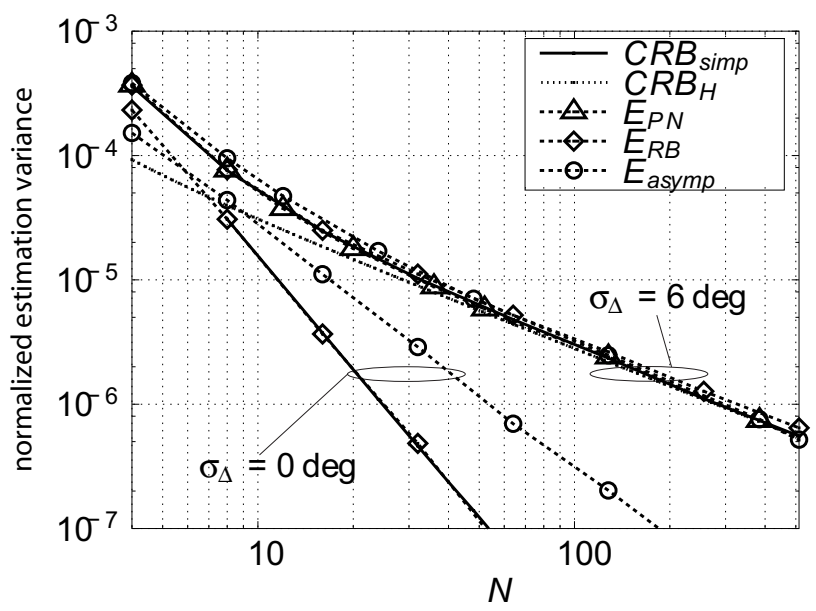

Fig. 5. Normalized estimator variance for $E_{S} / N_{0}=10 \mathrm{~dB}$ and $\sigma_{\Delta}=6$ degrees.

$\mathrm{CRB}$, as a function of the phase noise standard deviation for $E_{S} / N_{0}=10 \mathrm{~dB}$ and $N=64$ consecutive pilot symbols.

Finally, in Fig. 5, for $E_{S} / N_{0}=10 \mathrm{~dB}, L=1, \sigma_{\Delta}=0$ and 6 degrees, we show the performance of the estimators as a function of the number of pilots $N$. The $E_{P N}$ estimator is not reported for $\sigma_{\Delta}=0$, since in this case it exactly coincides with R\&B. We may observe that in the presence of phase noise, the normalized error variance decreases as $N^{-1}$ as predicted by the high-SNR asymptote of the CRB.

\section{CONCLUSIONS}

In this paper, the Cramer-Rao lower bound for frequency estimation in the presence of phase noise has been computed. Although it is not possible to derive a closed-form expression, we have shown an approximation that leads to a simple, fast, but very accurate evaluation of the bound by using a Monte Carlo average. The asymptotic closed-form expressions of the bound for low and high values of signal-to-noise ratio have been also provided. These expressions are very useful to better understand the effects of the phase noise on the frequency offset estimation accuracy. In particular, we demonstrated that in the presence of the phase noise it is not possible to reach the desired estimation accuracy by simply increasing the signal-tonoise ratio. These asymptotic expressions of the bound allow also to quantify the effect of the pilot distribution parameters and phase noise variance. Finally, a couple of ML-based algorithms specifically tailored for this scenario have been designed and compared with the best algorithm designed in the literature for the case of absence of phase noise.

\section{APPENDIX I}

\section{DERIVATION OF THE APPROXIMATED LIKELIHOOD FUNCTION}

From eqns. (4), (6a), and (6b), assuming $\rho(n)$ is small enough so as a single term in (7) can be considered, we have

$$
\begin{aligned}
& p(\mathbf{r} \mid \nu)=\frac{1}{2 \pi} \int \ldots \int t\left(z_{k(0)} ; \theta_{k(0)}\right) \prod_{n=1}^{N-1} t\left(z_{k(n)} ; \theta_{k(n)}\right) \\
& g\left(\theta_{k(n-1)}, \rho^{2}(n) ; \theta_{k(n)}\right) d \theta_{k(N-1)} \ldots d \theta_{k(0)}
\end{aligned}
$$

where we recall here the definitions of $z_{k(n)}$ and $\rho(n)$ :

$$
\begin{aligned}
z_{k(n)} & =\frac{r_{k(n)} a_{k(n)}^{*}}{\sigma^{2}} e^{-j 2 \pi \nu k(n) T} \\
\rho(n) & =\sigma_{\Delta} \sqrt{k(n)-k(n-1)} .
\end{aligned}
$$

Considering (9) and the approximation in (10), the integral in (B1) can be evaluated in a recursive manner.

1) First of all, we solve the innermost integral, obtaining

$$
\begin{aligned}
& \int g\left(\theta_{k(N-2)}, \rho^{2}(N-1) ; \theta_{k(N-1)}\right) \\
& \quad t\left(z_{k(N-1)} ; \theta_{k(N-1)}\right) d \theta_{k(N-1)} \simeq t\left(u_{N-2} ; \theta_{k(N-2)}\right)
\end{aligned}
$$

where we have defined the term $u_{N-2}$ as in (12).

2) Then, every integral, from the inner one to the outer one (but except the outermost), is of the form

$$
\int t\left(z_{k(n)} ; \theta_{k(n)}\right) t\left(u_{n} ; \theta_{k(n)}\right) p\left(\theta_{k(n)} \mid \theta_{k(n-1)}\right) d \theta_{k(n)}
$$

and it can be computed in a recursive manner, using (9), (10), and the definition (12).

3) The last (outermost) integral is of the form

$$
\begin{gathered}
\int t\left(z_{k(0)} ; \theta_{k(0)}\right) t\left(u_{0} ; \theta_{k(0)}\right) \frac{1}{2 \pi} d \theta_{k(0)} \\
=\frac{1}{(2 \pi)^{2}} \frac{\mathrm{I}_{0}\left(\left|z_{k(0)}+u_{0}\right|\right)}{\mathrm{I}_{0}\left(\left|z_{k(0)}\right|\right) \mathrm{I}_{0}\left(\left|u_{0}\right|\right)} .
\end{gathered}
$$

Neglecting the terms independent of $\nu$, the likelihood function shown in Section III results.

\section{APPENDIX II}

\section{DERIVATION OF THE LiKELIHOOd FUnCTION IN AbSENCE OF THERMAL NOISE}

In the absence of thermal noise, if we know the carrier frequency and the instantaneous phase we exactly know the received sample. Hence, the pdf $p\left(r_{k} \mid \nu, \theta_{k}\right)$ is a Dirac delta function, i.e.,

$$
p\left(r_{k} \mid \nu, \theta_{k}\right)=\delta\left(r_{k}-a_{k} e^{j\left(\theta_{k}+2 \pi \nu k T\right)}\right) .
$$

Inserting this expression in (4) we obtain

$$
\begin{aligned}
p(\mathbf{r} \mid \nu) & =\int \ldots \int \prod_{n=0}^{N-1} \delta\left(r_{k}-a_{k} e^{j\left(\theta_{k(n)}+2 \pi \nu k T\right)}\right) \\
& p\left(\theta_{k(n)} \mid \theta_{k(n-1)}\right) d \theta_{k(n-1)} \ldots d \theta_{0} \\
& =\int \ldots \int \prod_{n=0}^{N-1} \delta\left(\theta_{k(n)}-\arg \left[r_{k(n)} a_{k(n)}^{*} e^{-j 2 \pi \nu k(n) T}\right]\right) \\
& p\left(\theta_{k(n)} \mid \theta_{k(n-1)}\right) d \theta_{k(n-1)} \ldots d \theta_{0} .
\end{aligned}
$$

Now, remembering (7) and noting that $p\left(\theta_{k(0)} \mid \theta_{k(-1)}\right)=$ $p\left(\theta_{k(0)}\right)=\frac{1}{2 \pi}$, from $(\mathrm{C} 2)$ it follows that

$$
\begin{aligned}
p(\mathbf{r} \mid \nu) & =\prod_{n=1}^{N-1} p\left(\theta_{k(n)}-\theta_{k(n-1)}\right) \\
& =\prod_{n=1}^{N-1} \sum_{m=-\infty}^{\infty} g\left(2 \pi m, \rho^{2}(n), \theta_{k(n)}-\theta_{k(n-1)}\right) .
\end{aligned}
$$

Since for the CRB evaluation the likelihood function $p(\mathbf{r} \mid \nu)$ around its maximum nearest to $\nu=0$ has to be considered, 
for each $n$ only the term with $m=0$ has to be taken into account, while all the others with $m \neq 0$ are negligible for all the interesting values of $\sigma_{\Delta}$.

At this point, considering that if $z_{1}$ and $z_{2}$ are complex numbers, $\arg \left(z_{1}\right)-\arg \left(z_{2}\right)=\arg \left(z_{1} z_{2}^{*}\right)$, we can derive

$$
\begin{aligned}
p(\mathbf{r} \mid \nu) \propto & \prod_{n=1}^{N-1} \exp \left\{-\frac{1}{2 \sigma_{\Delta}^{2}[k(n)-k(n-1)]}\right. \\
& {\left[\arg \left(r_{k(n)} a_{k(n)}^{*} e^{-j 2 \pi \nu k(n) T}\right)\right.} \\
& \left.\left.-\arg \left(r_{k(n-1)} a_{k(n-1)}^{*} e^{-j 2 \pi \nu k(n-1) T}\right)\right]^{2}\right\} \\
= & \exp \left\{-\frac{1}{2 \sigma_{\Delta}^{2}[k(n)-k(n-1)]} \sum_{n=1}^{N-1}\right. \\
& {[2 \pi(k(n)-k(n-1)) \nu T} \\
& \left.-\arg \left(r_{k(n)} a_{k(n)}^{*} r_{k(n-1)}^{*} a_{k(n-1)}\right]^{2}\right\} .
\end{aligned}
$$

Finally, expanding the square, discarding some irrelevant constants and with simple manipulations

$$
p(\mathbf{r} \mid \nu) \propto \exp \left\{-\frac{(2 \pi)^{2}(k(N-1)-k(0))}{2 \sigma_{\Delta}^{2}}(\nu T-\hat{\nu} T)^{2}\right\}_{(\mathrm{C} 3 a)}
$$

with

$$
\hat{\nu} T=\frac{\sum_{n=1}^{N-1} \arg \left(r_{k(n)} a_{k(n)}^{*} r_{k(n-1)}^{*} a_{k(n-1)}\right)}{2 \pi(k(N-1)-k(0))} .
$$

\section{APPENDIX III}

\section{IMPLEMENTATION OF THE "FULL" MONTE CARLO Average Method}

The computation of the $C R B_{M C}$ can be decomposed into the following problems.

a) Let $\nu$ be the true value of the frequency offset. For a given received vector $\mathbf{r}$, the pdfs $p(\mathbf{r} \mid \boldsymbol{\theta}, \nu), p(\mathbf{r} \mid \boldsymbol{\theta}, \nu+\Delta)$, and $p(\mathbf{r} \mid \boldsymbol{\theta}, \nu-\Delta)$, for a value of $\Delta$ sufficiently small, are computed by generating a set of $N_{\text {int }}$ phase sequences $\boldsymbol{\theta}$ according to the Wiener model (2). For each of them, the pdfs $p(\mathbf{r} \mid \boldsymbol{\theta}, \nu)$, $p(\mathbf{r} \mid \boldsymbol{\theta}, \nu+\Delta)$, and $p(\mathbf{r} \mid \boldsymbol{\theta}, \nu-\Delta)$ are evaluated in closed-form following (6a). Then, according to (4), these $N_{i n t}$ values are arithmetically averaged. This step can obviously be avoided if we use the approximated closed form (11).

b) The second derivative necessary to compute (3) is approximated as

$$
\begin{aligned}
-\frac{\partial^{2}}{\partial \nu^{2}} \ln p(\mathbf{r} \mid \nu) & \simeq-\frac{1}{\Delta^{2}}[\ln p(\mathbf{r} \mid \nu+\Delta) \\
& +\ln p(\mathbf{r} \mid \nu-\Delta)-2 \ln p(\mathbf{r} \mid \nu)] .
\end{aligned}
$$

c) Finally, the expectation in (3) is evaluated by generating $N_{\text {ext }}$ sequences $\mathbf{r}$ following the channel model (1) and (2), the corresponding value of $-\frac{\partial^{2}}{\partial \nu^{2}} \ln p(\mathbf{r} \mid \nu)$ computed as described in the previous steps, and the $N_{\text {ext }}$ obtained values, arithmetically averaged to obtain the inverse of the CRB.

The step a) is crucial for the accuracy of the final CRB value, since to obtain a very accurate value of the second derivative $-\frac{\partial^{2}}{\partial \nu^{2}} \ln p(\mathbf{r} \mid \nu)$, the values of $p(\mathbf{r} \mid \nu), p(\mathbf{r} \mid \nu+\Delta)$, and $p(\mathbf{r} \mid \nu-\Delta)$ must be very accurate. Hence, it must be $N_{\text {int }} \gg N_{\text {ext }}$. The complexity of this "full" Monte Carlo average is roughly $N_{e x t} \cdot N_{\text {int }}$, where these two values have to be large enough in order to obtain a sufficient accuracy. For instance, after a proper tuning of these parameters we found that $N_{\text {ext }}=10^{5}$ and $N_{\text {int }}=5 \cdot 10^{6}$ give reliable results, as also confirmed by the perfect agreement with the lowand high-SNR expressions shown in the numerical results, and an increase in these values does not lead to different results. The use of the approximated closed-form likelihood function allows to avoid the inner expectation (4), thus roughly reducing the computational effort of a factor $N_{i n t}$. As shown in Section VI, there are no significant differences with respect to the results obtained by means of the "full" Monte Carlo method. Hence, this reduction of the computational effort comes "for free". The much lower simulation time allows, during design, a fast tuning of the system parameters, such as the number of pilots or their distribution.

\section{APPENDIX IV}

\section{LOW-SNR LIMIT OF THE CRB}

Let us consider the approximated likelihood function in (11). Since the coefficients $z_{k}$ are inversely proportional to the thermal noise variance and the case of low SNR is under consideration, coefficients $u_{n}$ can be considered small enough so as $\sigma_{\Delta}^{2}\left|u_{n+1}+z_{k(n+1)}\right| \ll 1$. Hence

$$
u_{n} \simeq u_{n+1}+z_{k(n+1)} \simeq \sum_{\ell=n+1}^{N-1} z_{k(\ell)} .
$$

Moreover, using the limiting form for small arguments $\ln \mathrm{I}_{0}(x) \simeq x^{2} / 4$, we find

$$
\begin{aligned}
\ln p(\mathbf{r} \mid \nu) & \simeq \frac{1}{4} \sum_{n=0}^{N-2}\left(\left|u_{n}+z_{k(n)}\right|^{2}-\left|u_{n}\right|^{2}\right) \\
& =\frac{1}{4} \sum_{n=0}^{N-2}\left[\left|z_{k(n)}\right|^{2}+2 \operatorname{Re}\left(z_{k(n)} \sum_{\ell=n+1}^{N-1} z_{k(\ell)}^{*}\right)\right]
\end{aligned}
$$

and after double differentiation with respect to $\nu$ we have

$$
\begin{aligned}
& \frac{\partial^{2}}{\partial \nu^{2}} \ln p(\mathbf{r} \mid \nu)=-\frac{(2 \pi T)^{2}}{2 \sigma^{4}} \sum_{n=0}^{N-2} \sum_{\ell=n+1}^{N-1} \\
& \quad \operatorname{Re}\left[r_{k(n)} r_{k(\ell)}^{*} a_{k(n)}^{*} a_{k(\ell)}(k(\ell)-k(n))^{2} e^{j 2 \pi \nu(k(\ell)-k(n)) T}\right] .
\end{aligned}
$$

In order to evaluate the CRB, (E1) has to be evaluated at the true value of $\nu$ and averaged with respect to the vector of the received samples. Noting that, for $n>m$,

$$
E\left\{r_{m} r_{n}^{*}\right\}=a_{m} a_{n}^{*} e^{-(n-m) \frac{\sigma_{\Delta}^{2}}{2}} e^{-j 2 \pi \nu(n-m) T}
$$

substituting this expression in (E1) and taking into account (3), we finally obtain

$$
\begin{aligned}
& C R B_{L}^{-1}=\frac{(2 \pi T)^{2}}{2 \sigma^{4}} \sum_{n=0}^{N-2} \sum_{\ell=n+1}^{N-1} \\
& \left|a_{k(n)}\right|^{2}\left|a_{k(\ell)}\right|^{2}[k(\ell)-k(n)]^{2} e^{-[k(\ell)-k(n)] \frac{\sigma_{\Delta}^{2}}{2}} .
\end{aligned}
$$




\section{REFERENCES}

[1] H. L. Van Trees, Detection, Estimation, and Modulation Theory - Part I. John Wiley \& Sons, 1968.

[2] D. C. Rife and R. R. Boorstyn, "Single tone parameter estimation from discrete-time observations," IEEE Trans. Inform. Theory, vol. 20, pp. 591-598, Sept. 1974.

[3] W. G. Cowley, "Phase and frequency estimation for PSK packets: bounds and algorithms," IEEE Trans. Commun., vol. 44, pp. 26-28, Jan. 1996.

[4] F. Rice et al., "Phase and frequency estimation for PSK packets: bounds and algorithms," IEEE Trans. Commun., vol. 49, pp. 1582-1591, Sept. 2001.

[5] N. Noels, H. Steendam, and M. Moeneclaey, "The true Cramer-Rao bound for carrier frequency estimation from a PSK signal," IEEE Trans. Commun., vol. 52, pp. 834-844, May 2004.

[6] S. M. Kay, "A fast and accurate single frequency estimator," IEEE Trans. Acoust., Speech, Signal Processing, vol. 37, pp. 1987-1990, Dec. 1989.

[7] M. Luise and R. Reggiannini, "Carrier frequency recovery in all-digital modems for burst-mode transmissions," IEEE Trans. Commun., vol. 43, pp. 1169-1178, Mar. 1995.

[8] U. Mengali and M. Morelli, "Data-aided frequency estimation for burst digital transmission," IEEE Trans. Commun., vol. 45, pp. 23-25, Jan. 1997.

[9] N. Noels, H. Steendam, and M. Moeneclaey, "Pilot-symbol assisted iterative carrier synchronization for burst transmission," in Proc. IEEE Int'l. Conf. Commun., Paris, France, June 2004, pp. 509-513.

[10] M. Morelli and U. Mengali, "Feedforward frequency estimation for PSK: a tutorial review," European Trans. Telecommun., vol. 9, pp. 103116, March/April 1998.

[11] S. Benedetto et al., "MHOMS: High speed ACM modem for satellite applications," IEEE Wireless Communications, vol. 12, pp. 66-77, Apr 2005.

[12] O. Macchi and L. Scharf, "A dynamic programming algorithm for phase estimation and data decoding on random phase channels," IEEE Trans. Inform. Theory, vol. 27, no. 5, pp. 581-595, September 1981.

[13] V. C. Vannicola and P. K. Varshney, "Spectral dispersion of modulated signals due to oscillator phase instability: White and random walk phase model," IEEE Trans. Commun., vol. 31, pp. 886-895, July 1983.

[14] U. Mengali and A. N. D'Andrea, Synchronization Techniques for Digital Receivers (Applications of Communications Theory). Plenum Press, 1997.

[15] L. Tomba, "On the effect of Wiener phase noise in OFDM systems," IEEE Trans. Commun., vol. 46, no. 5, pp. 580-583, May 1998.

[16] M. Peleg, S. Shamai (Shitz), and S. Galán, "Iterative decoding for coded noncoherent MPSK communications over phase-noisy AWGN channel," in IEE Proc. Commun., vol. 147, pp. 87-95, Apr. 2000.

[17] A. Demir, A. Mehrotra, and J. Roychowdhury, "Phase noise in oscillators: a unifying theory and numerical methods for characterization," IEEE Trans. Circuits and Systems I: Fundamental Theory and Applications, vol. 47, pp. 655-674, May 2000.
[18] A. N. D'Andrea, U. Mengali, and R. Reggiannini, "The modified Cramer-Rao bound and its application to synchronization problems," IEEE Trans. Commun., vol. 42, pp. 1391-1399, Feb. 1994.

[19] G. Colavolpe, A. Barbieri, and G. Caire, "Algorithms for iterative decoding in the presence of strong phase noise," IEEE J. Select. Areas Commun., vol. 23, no. 9, pp. 1748-1757, Sept. 2005.

[20] A. Barbieri, G. Colavolpe, and G. Caire, "Joint iterative detection and decoding in the presence of phase noise and frequency offset," in Proc. IEEE Int'l. Conf. Commun., vol. 1, May 2005, pp. 720-724.

[21] J. A. Gansman, J. V. Krogmeier, and M. P. Fitz, "Single frequency estimation with non-uniform sampling," in Proc. Asilomar Conf. Signals, Systems, Comp., Nov. 1997, pp. 399-403.

[22] A. Barbieri, G. Colavolpe, and G. Caire, "Joint iterative detection and decoding in the presence of phase noise and frequency offset," IEEE Trans. Commun., vol. 55, no. 1, pp. 171-179, Jan. 2007.

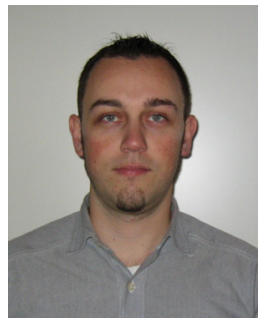

Alan Barbieri (S'05) was born in Parma, Italy, in 1979. He received the Dr. Ing. degree in Telecommunications Engineering (cum laude) from the University of Parma, Parma, Italy, in 2003. Currently, he is working towards the Ph.D. degree at Dipartimento di Ingegneria dell'Informazione (DII), University of Parma.

His main research interests include digital transmission theory and information theory, with particular emphasis on channel coding, iterative joint detection and decoding algorithms, estimation of unknown parameters and algorithms for synchronization. He participates in several research projects funded by the European Space Agency (ESAESTEC) and important telecommunications companies.

Mr. Barbieri was the recipient of the Premio Conti for the year 2003, as the best graduate in Information Engineering at the University of Parma in the academic year 2003 .

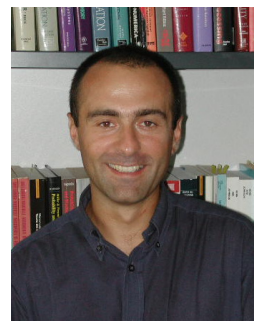

Giulio Colavolpe was born in Cosenza, Italy, in 1969. He received the Dr. Ing. degree in Telecommunications Engineering (cum laude) from the University of Pisa, Italy, in 1994 and the Ph.D. degree in Information Technology from the University of Parma, Italy, in 1998. Since 1997, he has been at the University of Parma, Italy, where he is now an Associate Professor of Telecommunications. In 2000, he was Visiting Scientist at the Institut Eurécom, Valbonne, France.

His main research interests include digital transmission theory, adaptive signal processing, channel coding and information theory. His research activity has led to more than eighty scientific publications in leading international journals and conference proceedings and a few industrial patents. He is also co-author of the book Detection Algorithms for Wireless Communications, with Applications to Wired and Storage Systems (New York: John Wiley \& Sons, 2004). In 2005, he received the best paper award at the 13th International Conference on Software, Telecommunications and Computer Networks (SoftCOM'05). 